\title{
Effect of filter structure on the filtration performance
}

\author{
Ying Jiang, ${ }^{1, \mathrm{a}}$, Ning Mao ${ }^{1, \mathrm{~b}}$,Jing Xian $\mathrm{Liu}^{1, \mathrm{c}}$ and Xi Sun ${ }^{1}$ \\ ${ }^{1}$ College of Resources and Civil Engineering, Northeastern University, \\ Shenyang,110004,China \\ a1143767872@qq.com, ${ }^{b}$ maoning@mail.neu.edu.cn, ${ }^{c}$ \\ liujingxian@mail.neu.edu.cn
}

\begin{abstract}
4 kinds of filter media with different structures are chosen and their filtration performance is comparative studied in the experiment. The relationships among filter resistance, flow velocity, aerosol concentration and efficiency are experimentally researched. The effect of filter structure on the filtration performance of filter media are discussed and deduced. It is found that some factors such as fiber diameter, filter pore size etc. have big influence on the filtration performance of filter media.
\end{abstract}

Keywords: filter media; filtration performance; efficiency; flow resistance.

\section{Introduction}

The rapid development of industry, promoting social progress, but also brought serious environmental pollution problems, a lot of smoke and toxic gas emissions into the atmosphere in the industrial production process, resulting in the concentration of suspended particles in the air severely exceed the standard, to produce the serious harm of air quality and human health. Air pollution is the focus of environmental pollution control in China, air filtration material is an important technique for atmospheric environmental governance, filter material efficient can exhaust the effective control of factories and enterprises emissions, reducing air pollution. The filtering properties of different media is the decisive factor of value and market prospects, efficiency and resistance is an important parameter to measure the filtration performance, so the efficiency and the resistance of filter test has important significance. ${ }^{[1-6]}$ 


\section{Experimental apparatus and materials}

Experimental apparatus TSI8130 automatic filter tester; 8118A aerosol generator (aerosol size on 0.3um); standard test paper; special red glass test filter; BT224S analytical balance; Sodium Chloride Solution 2\%.

Experimental materials Experimental tests of various material statistics in Table 1 , the scanning electron microscope is shown in figure 1.

Table 1The testing samples

\begin{tabular}{ccccc}
\hline Media number & $1 \#$ & $2 \#$ & $3 \#$ & $4 \#$ \\
\hline The filter & woven filter & non-woven felt & membrane felt filter & HEPA filter \\
\hline
\end{tabular}

By SEM can be seen from the picture, woven filter fiber diameter, arranged in a crisscross pattern, high porosity;non-woven felt about fiber aggregation, intertwined and cloth is a certain thickness and significant three dimensional form, the highest porosity;membrane felt filter material surface coated with a thin layer of plastic film, fiber are connected tightly, greatly reduce the porosity;comparison between HEPA fiber loose, diameter is the smallest, long and thin fibers, ratio of length to width,lower porosity.

\section{Results and discussion}

The relations between resistance and flow rate From Figure 2 we can know, various filter resistance increased with the increase of flow rate, and showed a significant linear relationship.Among them, membrane felt filter resistance with the increase of filtration velocity rise fastest, increased from $10 \mathrm{mmH}_{2} \mathrm{O}$ to $95 \mathrm{mmH}_{2} \mathrm{O}$, HEPA second, non-woven felt resistance increases the speed of the slowest, and the membrane felt filter resistance value is much higher than other media. 


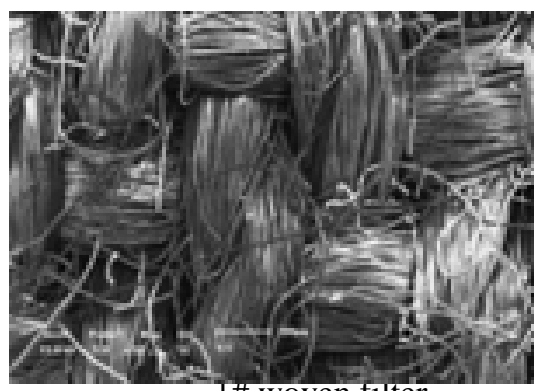

1\# woven filter

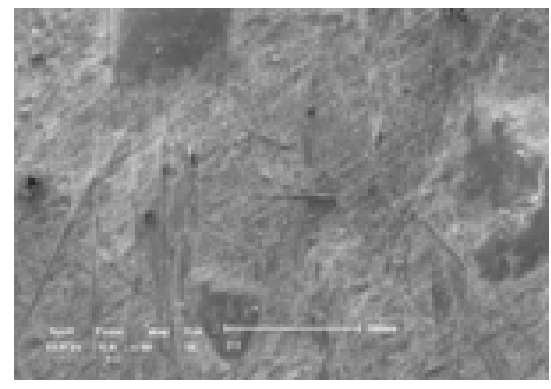

3\# membrane felt filter
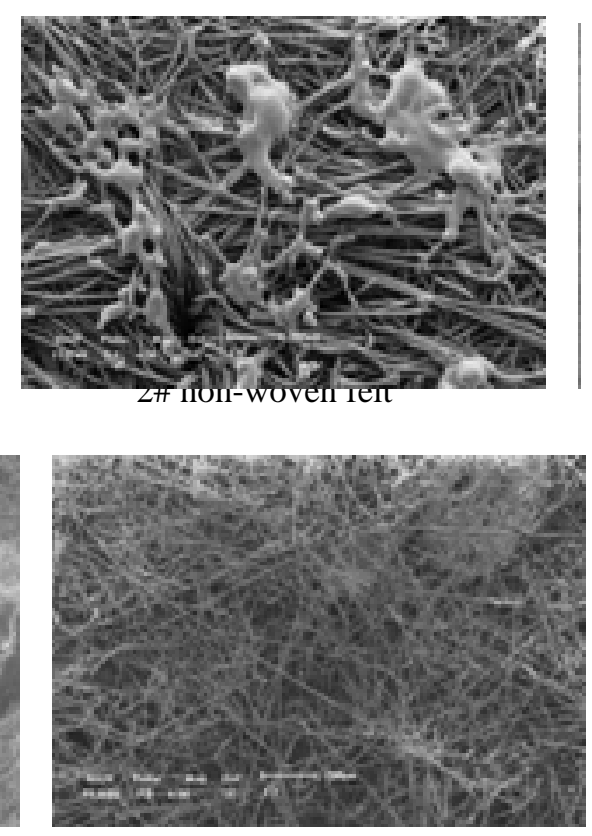

4\# HEPA filter

Figure 1 SEM photos of testing samples

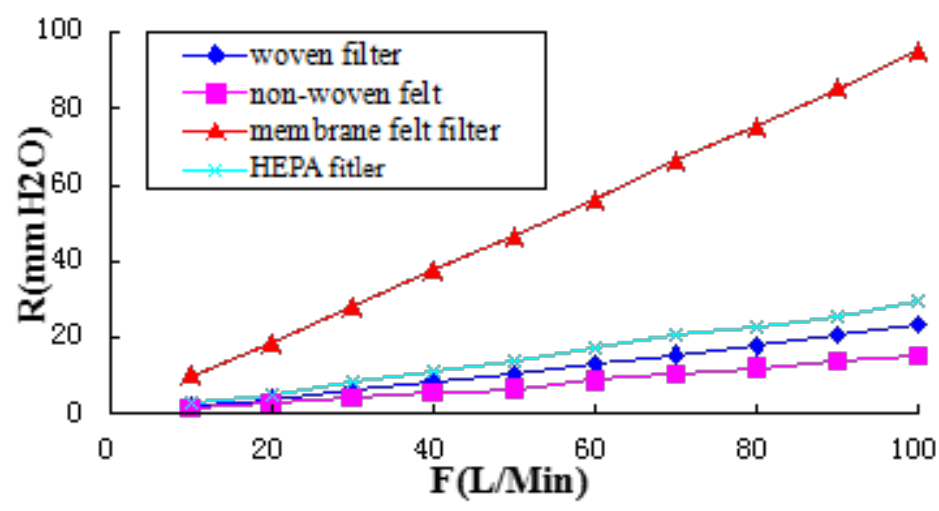

Fig. 2 Relationship between resistance and flow rate

The relationship between efficiency and flow rate Know from Figure 3,Membrane felt filter and HEPA have very high filtration efficiency, especially the efficiency of membrane felt filter can be stably maintained at between 
96\% 99\%, and with the increase of filtration rate of filtration efficiency throw will be a slight upward trend, the efficiency of other media with the filtration rate increase and drop in HEPA, which decreased the amplitude of the minimum, maximum amplitude decline in efficiency of non-woven felt, down from $47.2 \%$ to $25.2 \%$.

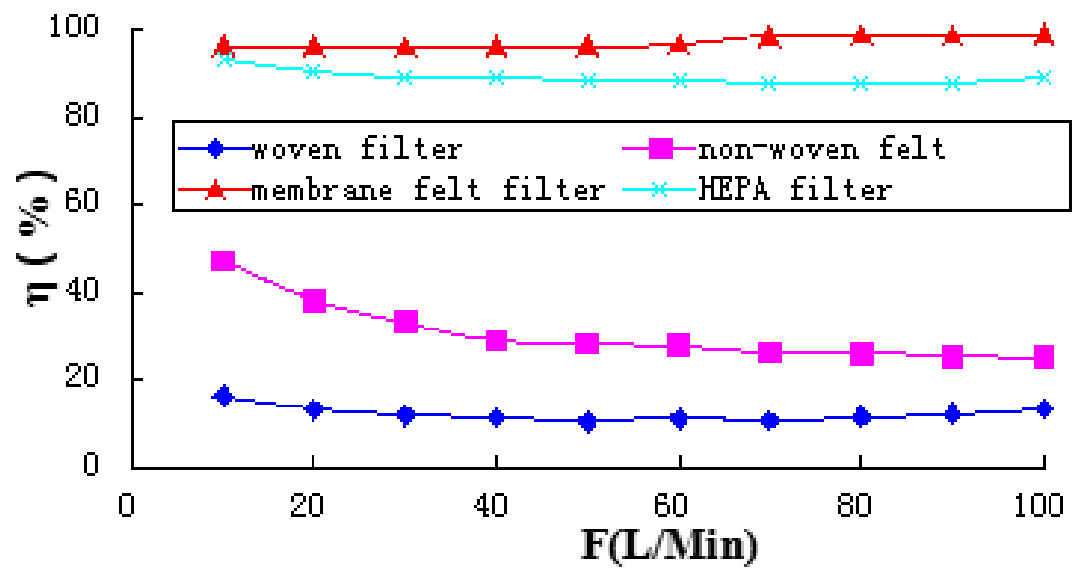

Fig. 3 Relationship between efficiency and flow rate

The relationship between efficiency and aerosol concentration Can see from Figure 4, with the increase of concentration, efficiency in the general media are showing a rising trend, in which membrane felt filter still maintained the highest efficiency, increased from $96.70 \%$ to $99.92 \%$, the efficiency of HEPA remained basically unchanged, the efficiency of non-woven felt increased the amplitude of the maximum, increased from $10 \%$ to $32.4 \%$.

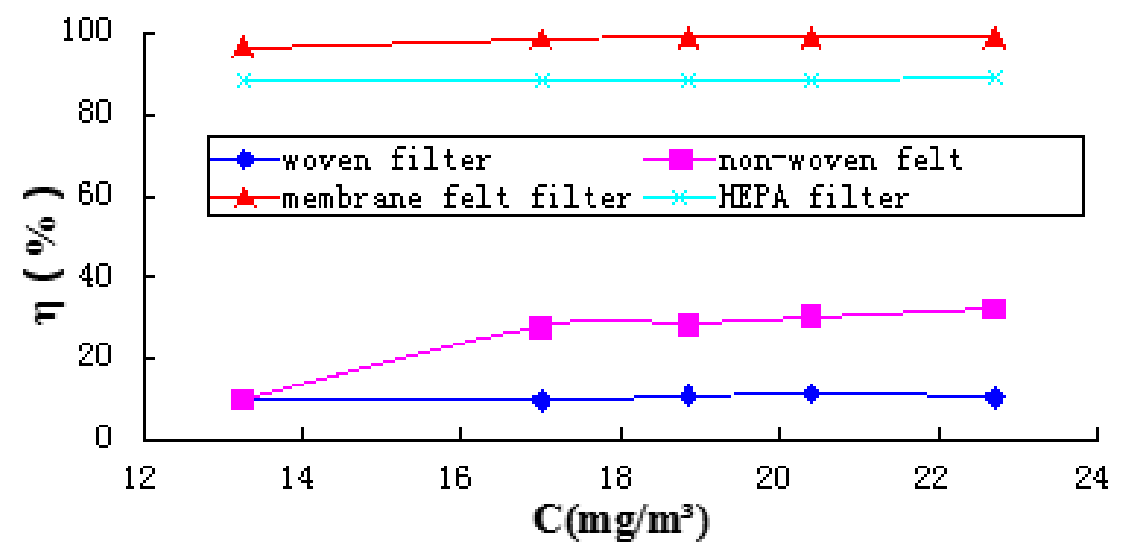


Fig. 4 Relationship between efficiency and mass concentration

The relationship between layers, the velocity and efficiency and resistance The relationship between layers, flow rate and efficiency Can know from Figure 5, when the filter efficiency increased layers of various filter will be increased significantly, membrane felt filter and HEPA filter from one layer to the two layer, efficiency has greatly improved the efficiency of felt, filter, membrane increased from $96 \%$ to $99.995 \%$, the efficiency of HEPA filter increased from $88.7 \%$ to $99.996 \%$, efficiency woven filter and non-woven of felt decreased with the increase of flow rate, and the efficiency of non-woven felt decreased the amplitude of the maximum.
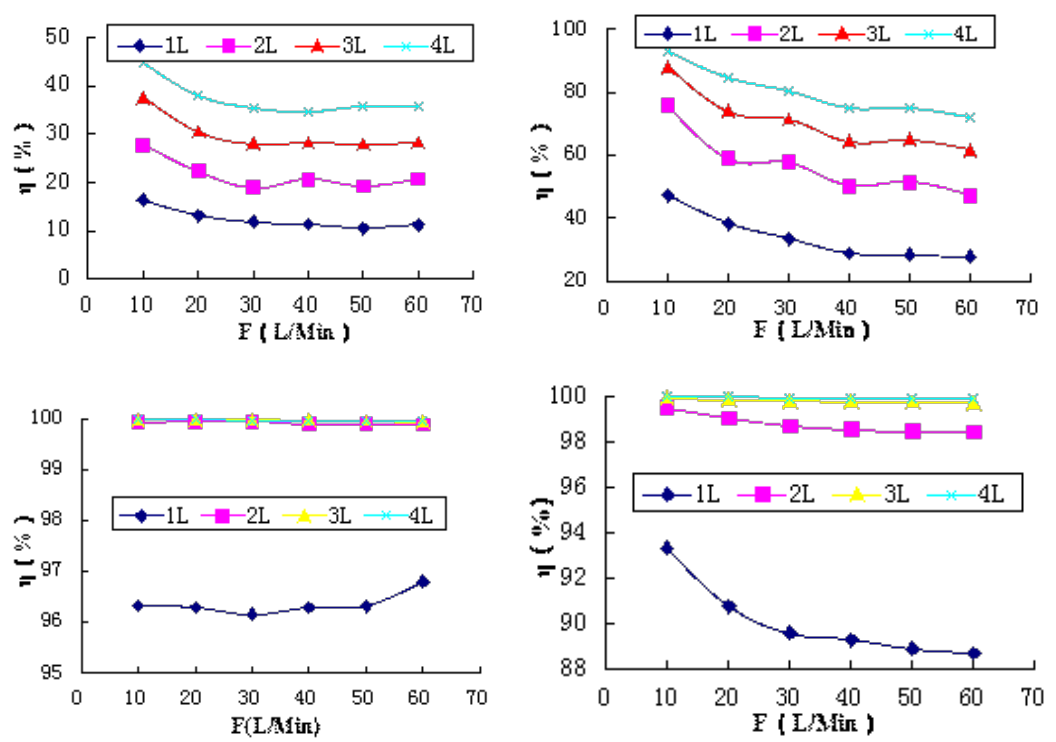

1\# woven filter $\quad$ 2\# non-woven felt $\quad 3 \#$ membrane felt filter

4\# HEPA filter

Figure 5 Relationship between the filter layer, efficiency and flow rate

The relationship between layers, flow rate and resistance We can see from Figure 6, the resistance of filter media with the increase of layers even resistance increases, and increases with increasing filtration velocity, it shows a linear relationship.The membrane felt filter resistance with the increase of flow rate, the rise in HEPAfilter second, non-woven felt resistance increase minimum. 


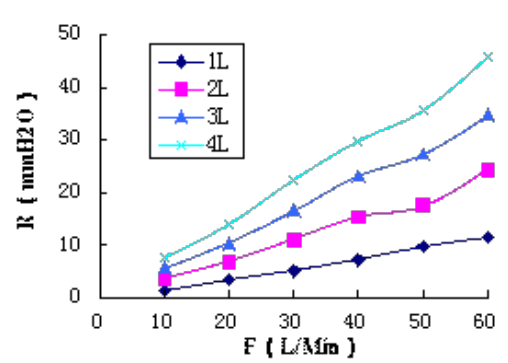

1\# woven filter

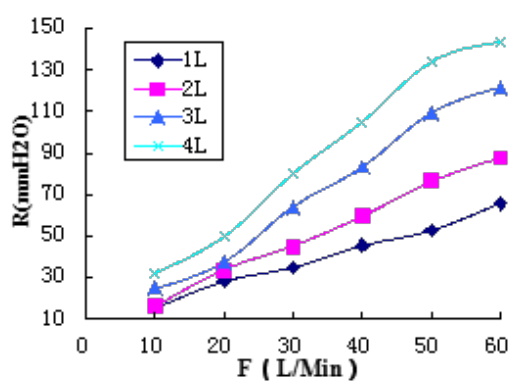

3\# membrane felt filter

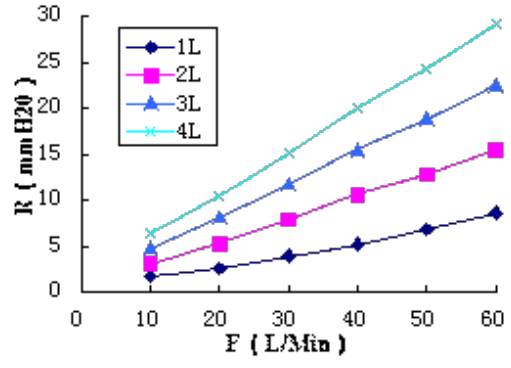

2\# non-woven felt

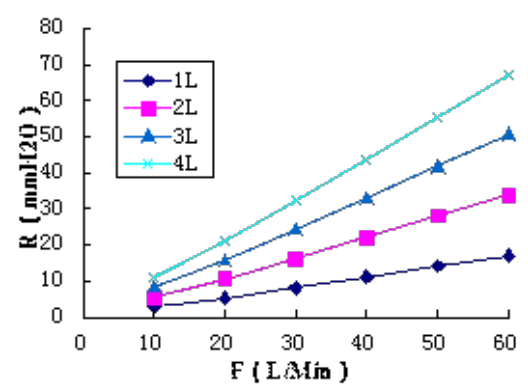

4\# HEPA filter

Fig. 6 Relationship between filter layer, resistance and velocity

\section{Conclusion}

Comprehensive experimental data can be seen, the filter of different structure has different efficiency value and resistance value under the same experimental conditions, so we can know the performance of structure and filter media are closely related, with all the experimental results can draw the following conclusions:

Woven filter belongs to the high resistance of inefficient compared with other media, the reason is because of its fiber crossing, poor permeability, porosity, fiber density is low, resulting in the filtration efficiency, and high resistance.

Non-woven felt because it adopts the mechanical consolidation method, the repeated puncture and forms its special structure, due to the presence of pinholes and the requirements for the fiber strength, the fiber diameter needle felt greatly, so its good air permeability, resistance with the increase of gas flow rate changed little, the filtering efficiency.

Membrane felt filter has the filtration efficiency and the highest resistance compared with traditional media, and the resistance is affected by the air flow is 
large, this is because of its fiber density, film and surface is covered with dense, plug the gap between fiber, low porosity permeability bad caused.

The efficiency of HEPA filter is higher than that of conventional media, slightly lower than the needle felt membrane filter material, and with the increase of air velocity increasing resistance value is lower than the needle felt membrane filter material, which is due to the fiber diameter of loose, small diameter, low porosity, good air permeability.

Comprehensive comparison of filter efficiency and the resistance value can know, compared with the conventional media, filtration efficiency of Non-woven felt and HEPA filter were significantly increased, while the HEPA filter permeability resistance value is small, but because of its fiber strength is not enough, the cost is higher practical problems, so its application is restricted, so the application of Non-woven on the market felt is the most widely.

Above all, we can know the different filter media fibre distribution, porosity, fiber diameter, fiber density, porosity, structure determines its different filtering performance, so to research and development of high performance material, should fully consider structural factors.

\section{Acknowledgement}

It is a project supported by the National Science and technology support program(2013BAC01B02) and the national high technology research and development program (863 Program) (2013AA065101).

\section{References}

[1] Jiang Ping, Liu Meihong.Development and application of air filtration materials [J].Modern textile technology, 2002,04:52-55.

[2] Huang Xueliang. High efficiency and low resistance, air filtration material preparation and Performance Research of [D]. Zhejiang Sci-Tech University, 2012

[3] Shen Zhiming The filter material of high performance and composite development[J].Nonwoven, 2005,04:9-12+23.

[4] Liu Chengkun, Ma Jianwei. The performance test and application of nonwovens filter materials [J].Nonwoven, 2005,01:30-34.

[5] Gao Feng. Study on filtering characteristics of fiber [D].Tianjin University, 2004 
[6] Zhang Cheng.The penetration rate of determination of the instantaneous pressure drop and the instantaneous filter TSI8130 automatic filter tester [J].China medical information, 2013,06:50-53. 